\title{
Modelo de innovación socio-institucional en la administración pública
}

\author{
Maria Victoria Sanagustin \\ Universidad de Zaragoza \\ vitico.sanagustin@gmail.com \\ Ignasi Brunet Icart \\ Universitat Rovira i Virgili \\ ignasi.brunet@urv.cat
}

Resumen: En este artículo se realiza una reflexión sobre cómo las administraciones públicas, consideradas como los instrumentos de aplicación de las politicas públicas, están aumentando su comportamiento transparente según ciertos indicadores y se propone un modelo de innovación socio-institucional para la implantación de un gobierno participativo, abierto y eficaz con control de los problemas de agencia en las organizaciones públicas, orientado al empoderamiento individual y social aprovechando la generalización de las tecnologías de la información y la comunicación, las redes sociales $y$ las organizaciones incidentales.

Palabras clave: Transparencia, gestión pública, teoría de la agencia moral, nuevo institucionalismo en sociología.

Abstract: In this article, a reflection is made on how public administrations are increasing their transparent behavior according to certain indicators and proposes a non-linear model of socio-institutional innovation for the implementation of an open and effective participatory government with control of the problems of Agency in the public administration, oriented to the individual and social empowerment taking advantage of the generalization of the technologies of the information and the communication, the social networks and the incidental organizations.

Key words: transparency, public management, theory of moral agency, new institutionalism in Sociology. 


\section{Introducción}

En este artículo se propone un modelo de innovación socio-institucional a partir de planteamientos que toman como punto de partida la promulgación de la Ley de la Transparencia en España y cómo los ciudadanos poseen una preocupación in crescendo por los casos denunciados y publicados de corrupción, así como una confianza social que anima a perseguir cotas mejores de eficacia y eficiencia en las administraciones públicas como ejecutoras de las políticas públicas. Asimismo, se describe el problema de agencia, basado en la teoría de la agencia moral aplicada a organizaciones de carácter público. Para ello, se hace una revisión teórica de los conceptos utilizados junto a la descripción de ciertos modelos posibles, estructurando el artículo en tres partes; primeramente, se plantea la tesis sobre el cómo y el porqué de la trasparencia, la rendición de cuentas y las formas de gestión, cuasi superadas, conocidas como Nueva Gestión Pública(NGP). Seguidamente, como un cuestionamiento de lo anterior, en el día a día de ciertas organizaciones, se reflexiona sobre el problema de la agencia moral en las entidades públicas. Por último, se propone un modelo de innovación que toma elementos de las diferentes teorías.

El método que se sigue en este artículo contiene análisis documental y análisis de datos secundarios procedentes de fuentes secundarias publicadas por organismos públicos y privados; obteniendo también testimonios de portales y blogs de solvencia informativa relativos al tema. Así se combina la reflexión teórica procedente de fuentes académicas con los datos objetivos procedentes de encuestas y testimonios de informantes profesionales del ámbito objeto de estudio, en este caso se trata de la población española en general y de personal directa e indirectamente relacionado con las administraciones públicas de diferentes ámbitos y localizaciones geográficas.

\section{El proceso modernizador de la gestión pública en España}

En los años noventa del pasado siglo xx, la administración pública en España comienza un periplo de cambio, es la denominada nueva gestión pública (Andreu y Brunet, 2010); considerada ya como un paradigma consolidado e incluso superado, abre puertas a nuevos conceptos y formas de administración (RamírezAlujas, 2010, 2011a, 2011b). En años recientes se impone una lógica marcada por la transparencia, la rendición de cuentas (accountability ${ }^{1}$, término inglés por

1 Accountability es un término acuñado primeramente por Philippe C. Schmitter y Terry Karl (1991), fue refinado por el propio Schimitter (2004) y por otros autores como Andreas Schedler (1999); describiendo la 
el que se le conoce) y el denominado Gobierno abierto que abarca conceptos y prácticas que trascienden el acceso a la información y sus reportes, implicando la participación activa y colaborativa de la ciudadan; un proceso que empieza por las administraciones locales (Swianiewicz, 2002; Lizcaíno, 2013) y que poco a poco va extendiéndose a diputaciones, hospitales, ministerios, universidades y otras organizaciones del ámbito público y semipúblico (Gutiérrez, 2014; García-Altés y Argimon, 2016; Pertuzé, 2015; Márquez, 2015; Cotino, 2015). Algunos indicios de dicha situación son la publicación, entre otros, de informes de sostenibilidad, códigos de conducta, e incluso de los resultados de auditorías externas (Moneva, 2007).

Sin embargo, el cambio se materializa con la promulgación en España de la Ley 19/2013 de Transparencia, Acceso a la Información Pública y Buen Gobierno. Una ley que ha recibido halagos y críticas desde diversos ámbitos pero que en la actualidad marca un devenir claro hacia la modernidad como innovación de la práctica política, la gestión pública y el fortalecimiento democrático. Algunos testimonios corroboran precisamente este extremo. Testimonio $\mathrm{A}^{2}$ : “Tener por fin una Ley de Transparencia en España creo que, de partida, es una buena noticia. También es cierto que debería haber sido más ambiciosa, abarcando más entidades y, especialmente, obligaciones sobre qué información publicar" (Responsable de Gobierno Abierto en la Junta de Castilla y León, 2015). O bien el testimonio del responsable de una agencia de comunicación política, testimonio B: "La Ley de Transparencia de España que se acaba de aprobar es un buen comienzo para que los ciudadanos fiscalicen la actividad administrativa y de los gobiernos de nuestro país pero es una norma que nace con algunas carencias. Entre ellas el no reconocer el acceso a la información como un derecho fundamental o las dificultades de registro para acceder a la información que hay disponible, que tampoco es toda la que existe" (Consultor de Comunicación política y de Gobierno. Coordinador ACOP Andalucía, 2015). O bien el testimonio C: “...España abandonaba el club de los países sin ley de acceso a la información, en Europa era el único país de más de un millón de habitantes que no contaba con legislación de este tipo. El título II dedicado al buen gobierno entró en vigor al día siguiente de su publicación, haciéndolo un año después el título preliminar, el título I (referido a la publicidad activa y el derecho de acceso a la información pública) y el título III (referente al Consejo de Transparencia y

denominada accountability vertical (acceso al poder) y la accountability horizontal (calidad del ejercicio de dicho poder); asimismo, se identifican otros aspectos tres internos y uno externo: por arriba (gobiernos), por abajo (sociedad civil), por dentro (burócratas) y desde fuera (actores internacionales).

2 Testimonios obtenidos en <https://politicacomunicada.com/nota-especial-7-opiniones-sobre-la-ley-detransparencia-espanola/>. 
Buen Gobierno) para la Administración General del Estado. La disposición final novena otorga un plazo máximo de dos años para adaptarse a las obligaciones de la ley a Comunidades Autónomas y Entidades Locales" (Especialista en e-administración y Open Data Administración pública del ayuntamiento de Lorca, 2015).

Si bien la ley genera ese marco de referencia, el proceso de transformación resulta complejo tanto en su implantación como en el análisis de las causas que lo han motivado. En primer lugar, es necesario considerar que cualquier cambio en una organización debe estar totalmente asumido por los miembros de la misma para que realmente sea un cambio admitido y real (Costa y López, 2010), para ello es imprescindible que sea participado, comprendido y asumido por el personal de la organización en cuyo seno se va a implantar; un trabajo de sensibilización, información y formación debe ocupar un espacio y tiempos necesarios para que dicho proceso cale profundo y sea una realidad, especialmente en el seno de las administraciones cuyo personal, en la mayor parte de casos, posee un empleo público permanente, de por vida.

Por otro lado, la complejidad del cambio, su implantación y evaluación igualmente vienen marcadas por las razones más o menos explícitas de su puesta en marcha. Al analizarlo, se observa que la lógica del mercado, como presión externa a la organización (Husillos et alii, 2011) se impone a la gestión pública; resultando que, en ocasiones, los líderes y autoridades públicas en su afán por mantener su status quo y siendo conscientes que para conseguirlo deben aparentar cumplir con las reglas del juego limpio y mejorar su imagen, imponen de forma más o menos consciente dicha lógica, al igual que sucede en el ámbito empresarial, sin embargo, también se observa cómo emergen reacciones paradójicas incluso desde quiénes plantean dicha transformación, pues resulta difícil consensuar, adaptar y hacer coincidir los intereses particulares de algunos líderes, gestores o técnicos públicos con los intereses de la organización que, en el caso de las administraciones públicas, se consideran parte del bien común de la sociedad. Y aquí, al igual que en la empresa surge un problema de agencia cuyas consecuencias pueden resultar nefastas para la administración pública correspondiente.

Igualmente, el cambio se debe al propio devenir de las sociedades democráticas cuya dinámica aparece marcada por unas exigencias mayores de la sociedad civil, tal y como se relata en el testimonio D, el informante afirma: "La puesta en marcha del portal de transparencia de España supone un punto de partida más que una estación de llegada. España llega con gran retraso en la aprobación de una ley de transparencia respecto de la mayoría de los países de su entorno. Qui- 
zás por ello, existe una mayor exigencia por parte de los ciudadanos para lograr una gobernanza abierta y participativa" (Cofundador de la red social de administración pública NovaGob, 2015); las autoridades públicas y los responsables técnicos (funcionarios de carrera) deben responder a las exigencias in crescendo de una ciudadanía cada vez más informada y conocedora de sus derechos (Subirats et al, 2009). La opinión de los europeos respecto a la confianza depositada en las instituciones está aumentando ligeramente en las últimas mediciones, lo que corrobora la idea de la respuesta correcta de dichas instituciones a las demandas señaladas, tal y como se comprueba en la imagen 1 .

\section{Imagen 1: Confianza de los europeos en las instituciones}

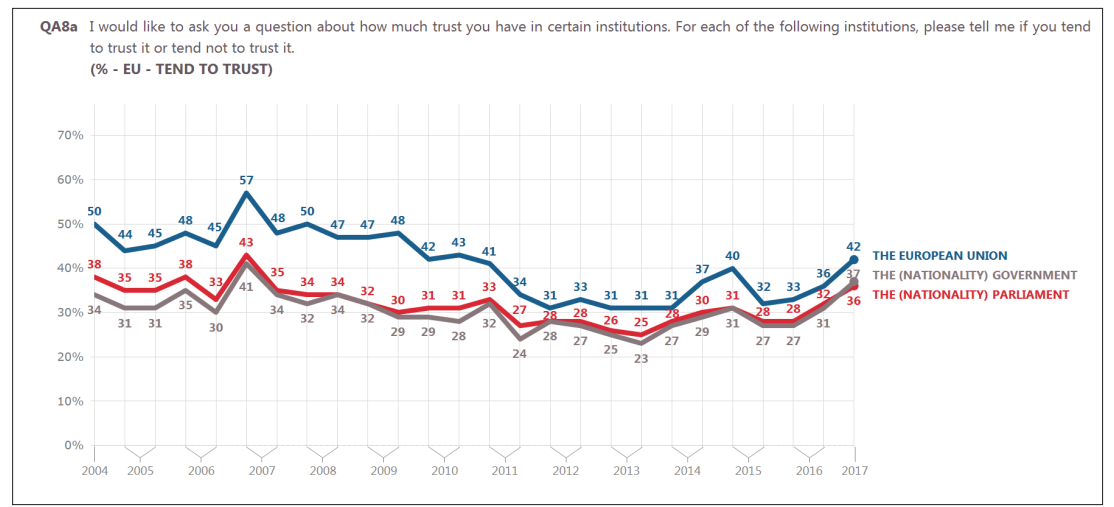

Fuente: Eurobarómetro, junio 2017

Además, la democracia española ya consolidada, cuyos fundamentos y constitución vienen acelerados por la globalización y el desarrollo de las tecnologías de la información y la comunicación demanda dicha transformación. La lógica del cambio y de la eficiencia que desde la ciencia económica se venía imponiendo a la gestión empresarial, se deriva y extiende a la gestión pública incluyendo la creación de redes interorganizacionales para la generación de políticas públicas (Lizcaíno, 2013; López y Rodrigo, 2014). Estas últimas razones descritas se pueden considerar como un cambio, en parte, inducido y no impuesto, precisamente por que tanto los usuarios de muchos de los servicios públicos como los propios funcionarios y empleados gubernamentales pertenecen a la sociedad civil. Una sociedad cada día más consciente de la obligación que el Estado, en sus diferentes formas y manifestaciones, posee respecto a la satisfacción de las necesidades de todos sus ciudadanos que además son considerados y se consideran a sí mismos contribuyentes y reclaman rigor y excelencia (Pérez Díaz, 1993; Subirats, 1999); la administración pública es el instrumento que facilita dichos servicios y debe responder de forma correcta (Pertuzé, 2015). 
Transparencia Internacional (2017), ONG independiente que analiza a través de indicadores institucionales el grado de transparencia de las corporaciones públicas en el mundo, también analiza la situación en España. En la imagen 2 se observa la evolución de las diferentes áreas de transparencia analizadas, dicha evolución ha ido in crescendo a lo largo de los últimos años, llegando al máximo de cumplimiento en el año 2017.

Imagen 2: Evolución en las áreas de transparencia en los ayuntamientos españoles

\begin{tabular}{|c|c|c|c|c|c|c|}
\hline ÁREAS DE TRANSPARENCIA & $\begin{array}{c}2017 \\
\text { (110 Aytos.) }\end{array}$ & $\begin{array}{c}2014 \\
\text { (110 Aytos.) }\end{array}$ & $\begin{array}{c}2012 \\
\text { (110 Aytos.) }\end{array}$ & $\begin{array}{c}2010 \\
\text { (110 Aytos.) }\end{array}$ & $\begin{array}{c}2009 \\
\text { (110 Aytos.) }\end{array}$ & $\begin{array}{c}2008 \\
\text { (100 Aytos.) }\end{array}$ \\
\hline TRANSPARENCIA GLOBAL: & 89,7 & 85,2 & 70,9 & 70,2 & 64,0 & 52,1 \\
\hline $\begin{array}{l}\text { A) TRANSPARENCIA ACTIVA E INFORMACIÓN } \\
\text { SOBRE LA CORPORACIÓN MUNICIPAL }\end{array}$ & 92,7 & 86,3 & 72,2 & 68,1 & 71,4 & 69,6 \\
\hline $\begin{array}{l}\text { B) PÁGINA WEB, RELACIONES CON LOS } \\
\text { CIUDADANOS Y LA SOCIEDAD, Y } \\
\text { PARTICIPACIÓN CIUDADANA }\end{array}$ & 90,7 & 86,8 & 76,3 & 77,3 & 71,4 & 69,0 \\
\hline C) TRANSPARENCIA ECONÓMICO-FINANCIERA & 93,1 & 90,0 & 71,2 & 63,8 & 49,1 & 29,1 \\
\hline $\begin{array}{l}\text { D) TRANSPARENCIA EN LAS CONTRATACIONES, } \\
\text { CONVENIOS, SUBVENCIONES Y COSTES DE } \\
\text { LOS SERVICIOS }\end{array}$ & 85,8 & 74,1 & 68,6 & 70,1 & 58,3 & 37,3 \\
\hline $\begin{array}{l}\text { E) TRANSPARENCIA EN MATERIAS DE } \\
\text { URBANISMO, OBRAS PÚBLICASY } \\
\text { MEDIOAMBIENTE }\end{array}$ & 86,0 & 85,8 & 77,6 & 72,2 & 67,0 & 48,4 \\
\hline F) INDICADORES LEY DE TRANSPARENCIA & - & 81,2 & 57,4 & - & - & - \\
\hline F) DERECHO DE ACCESO A LA INFORMACIÓN & 86,8 & - & - & - & - & - \\
\hline
\end{tabular}

Fuente: Transparencia Internacional, 2017

Los Ayuntamientos españoles que voluntariamente participan en el sondeo que realiza esta ONG anualmente, obtuvieron en 2016 una puntuación media de 89,7 sobre 100 en el conjunto de medidas, si bien son únicamente 25 los que obtienen la máxima calificación y únicamente 110 los que han participado activamente en la medición de los ochenta indicadores que incluye este control; lo que significa que queda bastante por hacer pero el cambio comenzado es imparable. Tal y como señala el propio informe, hay una serie de áreas pendientes en España, en las que se obtiene una menor puntuación, como son: la transparencia en las contrataciones ( $\left.85^{\prime} 8\right)$, y transparencia en materias de urbanismo y obras públicas $\left(86^{\circ} 0\right)$, siendo, por otro lado, las que reflejan una mayor puntuación las de: transparencia económico-financiera (93‘1), y transparencia activa (92‘7).

En la imagen 3 se comprueba la evolución de las medias de los ayuntamientos españoles en este sentido, una evolución en positivo, como se puede comprobar por los datos. 
Imagen 3: Evolución de la transparencia en ayuntamientos (España)

\begin{tabular}{|c|c|c|c|c|c|c|c|}
\hline $\begin{array}{l}\text { RANKING } 2017 \\
\text { (110 Aytos.) }\end{array}$ & AYUNTAMIENTOS & $\begin{array}{c}2017 \\
\text { (Media: 89,7) }\end{array}$ & $\begin{array}{c}2014 \\
\text { (Media: 85,2) }\end{array}$ & $\begin{array}{c}2012 \\
\text { (Media: 70,9) }\end{array}$ & $\begin{array}{c}2010 \\
\text { (Media: } 70,2)\end{array}$ & $\begin{array}{c}2009 \\
\text { (Media: 64,0) }\end{array}$ & $\begin{array}{c}2008 \\
\text { (Media: } 52,1)\end{array}$ \\
\hline 1 & ALCOBENDAS & 100 & 100,0 & 100,0 & 98,8 & 97,5 & 77,6 \\
\hline 1 & ALICANTE & 100 & 73,8 & 52,5 & 57,5 & 73,8 & 59,4 \\
\hline 1 & BARAKALDO & 100 & 92,5 & 97,5 & 85,0 & 35,0 & 61,9 \\
\hline 1 & BARCELONA & 100 & 100,0 & 88,8 & 88,8 & 90,0 & 81,3 \\
\hline 1 & BILBAO & 100 & 100,0 & 100,0 & 100,0 & 97,5 & 90,6 \\
\hline 1 & GETXO & 100 & 100,0 & 97,5 & 92,5 & 80,0 & 49,4 \\
\hline 1 & GIJÓN & 100 & 98,8 & 100,0 & 100,0 & 97,5 & 82,5 \\
\hline 1 & GIRONA & 100 & 72,5 & 77,5 & 73,8 & 72,5 & 63,1 \\
\hline 1 & HUELVA & 100 & 80,0 & 20,0 & 40,0 & 36,3 & 32,5 \\
\hline 1 & JEREZ DE LA FRONTERA & 100 & 95,0 & 51,3 & 86,3 & 60,0 & 55,0 \\
\hline
\end{tabular}

Fuente: Transparencia Internacional, 2017

Los indicadores de transparencia demuestran la lógica dominante en este terreno donde se manifiesta que la aplicación de la ley se está llevando a cabo de forma cada vez más generalizada en todas las administraciones públicas. En el informe de la consultora KPMG realizado en 2016 respecto a la aplicación de la ley de la transparencia en las diferentes organizaciones públicas en España, destacan algunos de los siguientes datos; en la imagen 4 , se observa que en un $79 \%$ de las organizaciones se han tomado diferentes medidas respondiendo a la entrada en vigor de la Ley de Transparencia.

Imagen $4: \vdots$ Se han tomado en su organización algún tipo de medidas como consecuencia de la entrada en vigor de la Ley de Transparencia?

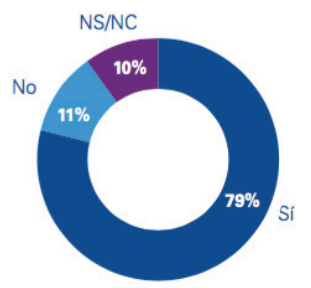

Fuente: KPMG, 2016.

Asimismo, se considera en dicho informe que el tipo y el grado de implantación de la transparencia en las administraciones públicas españolas, depende tanto del tamaño de la organización concreta, así como del tipo correspondiente. Tal y como se comprueba en la imagen 5. 
Imagen $5:$ ¿Cómo considera que se está efectuando la implantación de la Ley de Transparencia?

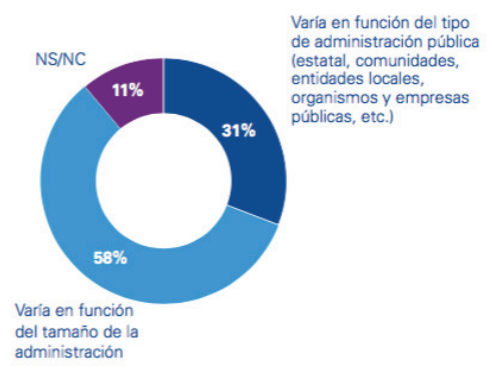

Fuente: KPMG, 2016.

Además, los datos procedentes del CIS en 2017 (Centro de Investigaciones Sociológicas) corroboran la tendencia social en España que plantea precisamente la corrupción como un problema prioritario al que hay que buscar soluciones; en su último barómetro, sondeo multirrespuesta expresadas en porcentajes (Junio, 2017), se muestra cómo los principales problemas percibidos por los españoles y por este orden, son: la corrupción y el fraude $(49,1 \%)$, los problemas de índole económica $(21,7 \%)$ y los políticos en general, los partidos políticos y la política (20,9\%); asimismo, la encuesta de Transparency International (2016) señala que 9 de cada 10 personas están dispuestas a actuar contra la corrupción y dos tercios de los encuestados se habían negado a pagar un soborno. Como consecuencia de los datos cabe señalar la demanda de los ciudadanos a un uso riguroso, limpio y eficaz de los fondos públicos en todos los ámbitos de la administración estatal, autonómica y local y en los diferentes entes públicos. La corrupción siendo una construcción sociocultural, no es irreversible (Cervini, 2016). En la imagen 6 se observa el nivel de confianza social agregada que los europeos ponen en los demás; España, se sitúa en un puesto medio-alto, lo que significa exactamente que la corrupción preocupa pero no llega a considerarse como un fenómeno generalizado, es decir existe confianza social, lo cual es positivo, dado que provoca optimismo social que empuja y activa a la ciudadanía a participar y colaborar, además, la confianza social genera felicidad. 
Imagen 6: "Se puede confiar en la mayor parte de la gente"

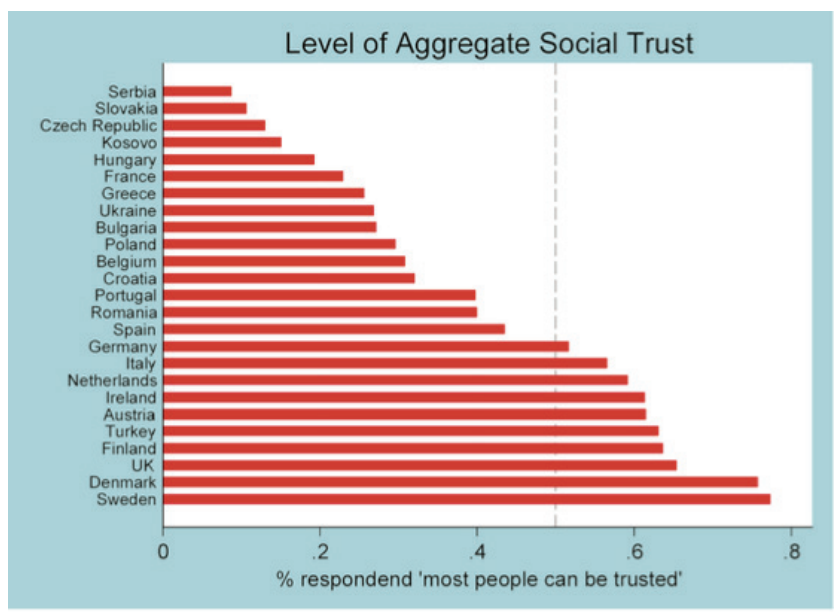

Fuente: Controlling Corruption in Europe. The Anticorruption Report, 2013.

En la Imagen 7 se pueden observar datos reveladores procedentes de la encuesta europea sobre corrupción y gobernanza. Las escalas de azules más oscuros significan que en las regiones de ese color la percepción respecto a la corrupción es menor y dicho gradiente va aumentando conforme el color se hace más rojo y oscurece. España muestra todas las regiones en tonos azules y únicamente son cinco de las diecisiete comunidades autónomas las que tienen un color rojo muy suave y Galicia un poco más fuerte. Lo que corrobora, de nuevo, los argumentos sobre cómo en España la corrupción preocupa y el proceso de cambio muestra el aumento de las denuncias y cómo se publican los casos, aplicando sobre ellos la ley y la justicia del estado de derecho (Gutiérrez, 2014; Lizcaíno, 2013). 
Imagen 7: Mapa sobre percepción de la corrupción

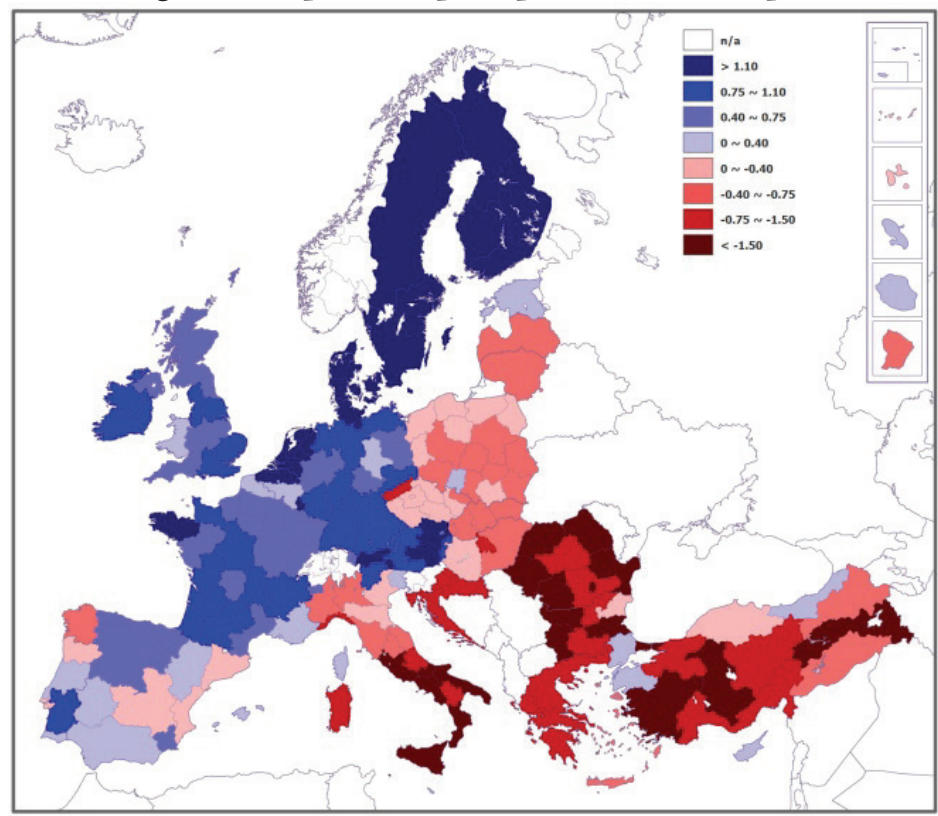

Fuente: European Quality of Government Index (EQI), Charron 2013.

Los principales hitos y procesos que han provocado la transformación hacia la aplicación de la transparencia, la rendición de cuentas y el gobierno abierto, en la historia reciente de España y del resto de países occidentales, coinciden con la propia evolución de las teorías del management dentro y fuera de las administraciones públicas y se recogen en la siguiente tabla.

\section{Tabla 1: Hitos y procesos relevantes en la implantación de cambios en la gestión pública}

\begin{tabular}{|l|l|}
\hline Hitos y procesos relevantes & Consecuencias en la gestión pública \\
\hline $\begin{array}{l}\text { EFQM (European Framewok for Quality } \\
\text { Management) }\end{array}$ & Calidad Total, el usuario se convierte en cliente \\
\hline Desarrollo y generalización de las TICs & $\begin{array}{l}\text { Democratización del conocimiento y acceso a la información de } \\
\text { manera global }\end{array}$ \\
\hline Globalización & Mercado global \\
\hline $\begin{array}{l}\text { Business Etbics, Responsabilidad social } \\
\text { corporativa (RSC) y Desarrollo Sos- } \\
\text { tenible }\end{array}$ & $\begin{array}{l}\text { Yolíticas responsables desde la triple perspectiva social, económica } \\
\text { el público }\end{array}$ \\
\hline $\begin{array}{l}\text { Planificación estratégica } \\
\text { Crisis económica mundial, 2008 }\end{array}$ & $\begin{array}{l}\text { Búsqueda de la eficiencia dados el aumento de la conciencia de los } \\
\text { recursos limitados en las administraciones públicas }\end{array}$ \\
\hline $\begin{array}{l}\text { NIS (Nuevo Institucionalismo en So- } \\
\text { públicas en España }\end{array}$ & $\begin{array}{l}\text { Legitimación de las organizaciones. Isomorfismo. Rendición de } \\
\text { cuentas }\end{array}$ \\
\hline
\end{tabular}

Fuente: Elaboración propia. 
El primero de los hitos tiene que ver con la gestión de la calidad total (GCT) o TQM (Total Quality Management) procedente de Estados Unidos y Japón y que en Europa se desarrolló como modelo EFQM (European Framework for Quality Management). Buscando la calidad y la excelencia de los servicios públicos, aquello que nació en la industria norteamericana y japonesa se extendió rápidamente a la industria europea y, casi de forma cronológica y seguida, a los servicios públicos de un cada vez mayor número de países occidentales y principalmente en Europa (Carbellido, 2005).

El segundo, con la extensión de la Business Ethics (Sanagustín, 2011) que actualmente se utiliza como instrumento de rendición de cuentas y que se desarrolla a través de los procesos voluntarios empresariales de responsabilidad social junto al concepto extendido y determinante del desarrollo sostenible. Existen movimientos sociales globales que abogan por la conservación del medio ambiente, dicho objetivo se ha convertido en el leit motiv de diferentes cumbres mundiales por el medio ambiente y más recientemente, los objetivos del milenio y así lo reconoció Naciones Unidas y sus 193 Estados miembros en 2015 al aprobar la Agenda 2030 para el Desarrollo Sostenible.

El tercer hito, parte de los conceptos y procesos desarrollados ampliamente en el mercado y las empresas como son los que se conocen como planificación estratégica, dichos procesos son una forma de asegurar y garantizar que los fondos públicos invertidos en determinadas administraciones públicas se utilizan adecuadamente, con los objetivos correctos y gestionando de la mejor forma posible. En este planteamiento el entorno constituye uno de los factores determinantes para la toma de decisiones en las administraciones, orientando acciones, esfuerzos y presupuestos a alcanzar metas que se diseñen en función de dicha variable.

Otro de los hitos que han marcado la evolución reciente de la gestión de las administraciones públicas es la crisis económica mundial de 2008. Observado como uno de los acontecimientos más recientes, pero no menos importantes de la última década (Mason, 2015), surgida precisamente por el desmantelamiento de Lehman Brothers en USA; la consultora había vendido sin control y globalmente las acciones basadas en hipotecas basura millonarias y todo eso se volvió en contra del propio sistema, generando un agujero irreparable cuyas consecuencias todavía están presentes en la economía mundial; una sucesión de acontecimientos relacionados con dicha estafa se desencadenan en el mundo, otra de las más importantes consultoras británicas Standard \& Poor's se encuentra en la misma situación, su directora general admite haber estado vendiendo humo durante años. El caos financiero repercute en el sistema económico global, Europa y España por ende, comienzan a sumirse en una crisis económica y financiera sin 
precedentes que en nuestro país se ve agudizada por que la burbuja inmobiliaria estalla y el desempleo estructural aumenta descontroladamente (Castells, 2014); así el gobierno del presidente José Luís Rodríguez Zapatero que durante varios meses negaba la sombra de la recesión económica se ve obligado a generar más deuda pública de la prevista, encontrándose además la deuda privada tanto de los hogares como del sector empresarial y especialmente bancario y en unos niveles excesivamente altos. Así comienza una etapa difícil para España, cambia el gobierno y el PP que gana las elecciones en 2012, realiza una readaptación de los presupuestos públicos y comienza una etapa de recortes, imponiendo una cultura de la austeridad la cual llega a la mayor parte de administraciones públicas. Se destapan escándalos financieros unidos al sector inmobiliario y también desfalcos en algunas administraciones públicas, no es raro leer y escuchar en los mass media noticias relacionadas con denuncias financieras en las que se imbrican tanto el ámbito privado como el público; banqueros en la cárcel, representantes políticos denunciados y pendientes de condenas y un largo etcétera de situaciones similares en la mayor parte de las regiones españolas (Keane y Feenstra, 2014).

Junto a la evolución de estos hitos o antecedentes históricos en la nueva gestión pública, se fragua la insistencia de la ciudadanía por conocer el acontecer de las instituciones financiadas por todos, así como poder determinar y definir políticas y programas que respondan a necesidades reales; igualmente, conocer el estado de cuentas de organizaciones no gubernamentales y de asociaciones de la sociedad civil que reciben subvenciones y ayuda voluntaria de mucha gente. El concepto procedente de la teoría conocida como nuevo institucionalismo en sociología plantea, entre otros, la rendición de cuentas como una forma de legitimar cualquier organización frente a la ciudadanía, algo que es imprescindible en una sociedad compleja, cada vez más informada y que exige precisamente un comportamiento correcto a las instituciones y entidades que dependen de ella.

El último hito descrito es el Nuevo Institucionalismo en Sociología que llega a calar hondo en la explicación organizacional actualmente tanto cuando se pone la mirada en la organización privada como, más recientemente, en la organización pública. Para legitimar a las organizaciones en la sociedad, se desarrolla un proceso denominado isomorfismo, consistente en la imitación de prácticas llevadas a cabo por empresas y organizaciones que están ya legitimadas socialmente y así, se da respuesta a las presiones sociales y del entorno, reproduciendo dichas prácticas llevadas a cabo por las que han logrado el éxito o la aceptación social (DiMaggio y Powell, 1983). Estos autores señalan al isomorfismo como la razón dominante por la que las organizaciones adoptan formas diversas. Parten de la 
hipótesis de considerar a la organización dentro de campos organizativos ( Scott, 1995), es decir en campos con organizaciones similares de reconocida vida institucional. Los tipos de isomorfismo son la respuesta a las presiones recibidas desde el entorno de las organizaciones; dicho isomorfismo se clasifica como coercitivo (característico de la administración pública respondiendo a presiones tanto formales como informales), mimético (respondiendo a una elevada incertidumbre y cuándo existe una organización exitosa en el campo imitable por parte de las demás) y normativo (relacionado con las normas sociales predominantes y a las que la organización se adapta e implanta). Así, las organizaciones se relacionan y establecen sinergias con los diferentes agentes sociales, económicos, políticos, culturales, y asumen responsabilidades en la sociedad en la que están inmersas (Wheeler y Sillanpää, 1997), todo ello modela su legitimidad social. $\mathrm{El}$ nuevo institucionalismo define las instituciones como sistemas que incorporan construcciones cognitivas y normativas en el seno de la sociedad en la que operan, incluyendo las propias administraciones públicas, basadas en estructuras reguladoras, cognitivas y normativas. Hoy, la administración pública española se orienta a responder a las demandas ciudadanas.

\section{La teoría de la Agencia, la prevalencia del interés individual por encima del interés de la organización y del bien común.}

La teoría de la agencia describe el funcionamiento de las organizaciones en relación al posible enfrentamiento de intereses entre ciertos individuos (agentes) que trabajan en la organización y los propietarios de la misma que se identifican con los intereses organizacionales (principal/es) (Jensen y Meckling, 1976 ). Jensen y Meckling (1998) consideran que ambos son diferentes y que el agente no siempre está orientado a velar por las garantías de la empresa, tal y como lo está el principal. Este problema de agencia, se puede trasladar a las administraciones públicas, de tal forma que el agente serían los empleados públicos y las propias autoridades que desempeñan su labor como representantes del Estado, el comitente o principal podría ser, o bien la propia sociedad que es a quien representa y sobre quién recae la acción del agente (Fidone, 2011), o bien, la entidad pública en su conjunto, entendida como organización en sus diferentes tipos, tamaños y cometidos. La literatura ha identificado el riesgo en la relación de agencia por la dificultad real de controlar si el agente está trabajando por el bien común o por sus propios intereses, es el riesgo moral (Hölmstrom, 1979), de ahí que se hable en muchas ocasiones del problema de la agencia moral. 
Dicho problema de agencia es frecuente en las administraciones/ entidades públicas, provocando ineficiencia, falta de control y baja rendición de cuentas. $\mathrm{El}$ agente posee poder, distinto al que acontece en la empresa privada, pero muy similar, pues no son pocos los casos de funcionarios de alto nivel o políticos retirados que terminan ocupando puestos en consejos de administración de compañías privadas.

Lo que en un principio podría parecer bondadoso en el sentido del concepto de eficiencia y de dar la posibilidad a la ciudadanía de tener un cierto control sobre el quehacer de la administración pública a través de una ley como la de la transparencia y su aplicación en las diferentes administraciones públicas; se convierte en un sistema perverso de una forma cuasi imperceptible como lo que acontece en las entidades privadas de las que se tomó el modelo. La teoría de la agencia moral identifica el poder acumulado por unos pocos, una persona o un grupo de unos pocos; describe además cómo la toma de decisiones del agente responde principalmente a sus intereses y de las dificultades a las que somete al denominado principal (la propia administración y/o la sociedad en su conjunto) en determinadas ocasiones y exclusivamente buscando su propio interés. Partiendo de la idea de que las organizaciones están construidas por personas, la teoría de la agencia (Jensen y Meckling, 1998) explica claramente cómo hay personas en las organizaciones empresariales que convierten la lucha por sus propios intereses en una batalla a la que someten a toda la organización. Este tipo de personas se pueden identificar también en organizaciones públicas ¿¿De qué tipo de intereses estamos hablando? Aquellos que emanan no solamente del dinero, si no también del poder por el poder, el prestigio, el control, la influencia y su tráfico, en definitiva lo que implica estar en una situación de superioridad respecto al resto de personas con el único afán de demostrarlo y perpetuarlo a cualquier precio y de cualquier forma. Existen innumerables ejemplos en los que ostentar un cargo, una jefatura, una responsabilidad en la gestión pública implica cierta discrecionalidad en el proceso de toma de decisiones; no se vulneran las leyes, ni el derecho pero existe un límite muy sutil entre lo correcto y lo incorrecto, entre lo moralmente reprochable y la ética. Por tanto, podrían surgir comportamientos escondidos del agente, que, sacando provecho del control insuficiente de su tarea por parte del principal (independientemente de con quién se identifique, la sociedad en su conjunto o quiénes son representantes de la misma a través de consejos sociales $u$ otros), puede perseguir su propio interés en lugar del comitente (Fidone, 2011).

Se pueden identificar diferentes modelos de situaciones en las que emerge un problema de agencia en las administraciones públicas. A continuación se describen cuatro. 
Modelo A: Asimetría en el manejo de la información y orientación ideológica. Cargo público que dirige una organización pública compleja (universidad, hospital, ...) orientando sus acciones y decisiones, gracias a toda la información de la que dispone, a apoyar al partido que gobierna en un momento determinado. El interés particular del agente no es incompatible con los objetivos organizacionales; pero, en esta situación imaginaria, se podría generar conflicto de intereses e incluso emerger un problema de agencia moral pues las decisiones tomadas están orientadas más al bien de unos pocos que al del conjunto de la sociedad o de la eficiencia de la propia organización.

Modelo B: Divergencias de intereses y competencial. Se trata de las dificultades existentes en los procesos de selección de ciertos mandos de las administraciones públicas que cumplen los requisitos exigidos pero luego, gracias a cierta discrecionalidad y opacidad de las normas permitidas, actúan bajo su propio interés, e incluso en algunos casos, sin la eficacia y competencia profesional exigidas.

Modelo C: Ausencia o fragilidad de los sistemas de control. El agente puede actuar en la organización pública o semipública con cierta discrecionalidad y, todavía en la actualidad, faltan mecanismos de control y transparencia en los diferentes niveles analizados: contratos, información y acceso a la misma, contabilidad analítica e informes de sostenibilidad, acceso a la toma de decisiones y al planteamiento de temas que interesan a la ciudadanía en su conjunto o al grupo social implicado en dicha organización.

Modelo D: Marcos regulatorios deficientes. Se puede considerar que existe una crisis de mecanismos de regulación, demostrando en la práctica que las normativas existentes son insuficientes y en muchas ocasiones inadecuadas en sus enfoques y en sus ámbitos de control, por lo que son rebasadas por los hechos (Conteras et alii, 2015).

El factor común en todos los modelos es el interés individual de quién comete los abusos que no se manifiestan en ningún momento como tales, si no que pasan desapercibidos por formar parte de la discrecionalidad que acompaña a determinados cargos dentro de las administraciones públicas; es decir, en ningún caso ha habido abuso de poder, en ningún caso hay denuncias, en ningún caso ha salido nadie dañado directa y manifiestamente. Pero en todas y cada una de las posibles situaciones hay un problema de agencia, un problema de abuso de poder de quién en un momento concreto tiene la capacidad de tomar decisiones cuyas consecuencias podrían generar un deterioro de la convivencia, la cohesión social e incluso un desmantelamiento y empeoramiento de la organización/ entidad pública o semipública en su conjunto y del bienestar social. Dado que en la toma de decisiones ha primado dicho interés particular por encima del interés de la 
organización y el bien común. Son entidades públicas, sin embargo, se observa un problema de agencia evidente.

Cada uno de los sujetos de una relación de agencia tiene sus propios intereses y ambos son maximizadores de utilidad. El problema del principal "es ver si existe algún sistema de recompensas para el agente que produzca una solución paretoeficiente para cualquier par de funciones de utilidad del agente y el principal" (Ross ,1973:69). Una de las partes más importantes de la teoría de la agencia es fundamentar el conocimiento y la información, la separación entre los derechos de propiedad y los derechos de decisión. Estos últimos implican delegar responsabilidad sobre las decisiones en el agente que las ejecuta.

El modelo expuesto no garantiza en la practica un control efectivo del problema de agencia porque ningún sistema de control puede encauzar de forma inequívoca el comportamiento de agentes a los cuales se les da un amplio margen de actuación y discrecionalidad en su toma de decisiones. También porque la idea misma de control tienen imperfecciones (Williamson, 2005). La solución del mismo requiere que se produzca una institucionalización de carácter social compleja, integral e integradora.

\section{Conclusión: Modelo de convivencia, innovación social e institucional}

$¿$ Es posible controlar e incluso penalizar los casos de agencia moral en las administraciones públicas? ¿poseen los ciudadanos herramientas asequibles, como las que emanan de la aplicación de la Ley 19/2013 de Transparencia, Acceso a la Información Pública y Buen Gobierno, para garantizar que las administraciones públicas gestionen de la mejor forma y se alcancen objetivos y metas deseados por la mayoría? Incluso, ¿se puede promover en España un Gobierno abierto eficaz y eficiente?

Se parte de la idea que la desafección política e institucional por parte de las sociedad civil en las democracias occidentales del siglo xxi (Requena y Rodríguez, 2017), y en la española en particular, es uno de los graves problemas sociales a los que se enfrenta el sistema político en general y por ende, la propia administración pública que es el instrumento que materializa la puesta en marcha de las políticas. Se observa además, que tras los innumerables casos de corrupción política, institucional y empresarial, el modelo que se propone parte de la aplicación de la Ley de la transparencia y del impulso de la rendición de cuentas (accountability) para garantizar el uso correcto de los fondos públicos, involucrando así 
a agentes sociales, económicos, políticos y a los propios funcionarios y técnicos responsables de las administraciones.

El modelo de innovación socio-institucional que se presenta es aplicable a entidades públicas, partiendo de un aprendizaje social e individual, en diferentes ámbitos para que mejoren su actuar y ello tenga como consecuencia que las personas jóvenes se animen a participar y colaborar activamente en política y en un espacio público de compromiso ciudadano. Este modelo es un instrumento para generar cambio y mejorar el sistema, considerando que dicho sistema no va a desaparecer pero debe regenerarse y mejorar; manejando ideas complejas que intentan salir del pensamiento dicotómico instaurado frecuentemente en las ciencias sociales. Asimismo, se parte de un concepto básico, el de norma perversa acuñado por Férnandez Dols en el año 1993, definiendo la misma como aquella que no la cumple la mayoría de la gente por haberse generado como norma impuesta no participada ni consensuada; dichas normas generan tolerancia a la corrupción y además no son deseadas, resulta evidente que no todas las normas y/o leyes pueden ser consensuadas, sin embargo, cabe reconocer que hay una cantidad de ellas que afectan directamente a la ciudadanía y que podrían y deberían ser mejor comunicadas y trabajadas. Como propuesta que caracteriza el modelo está el uso de la tecnología la cual forma parte de muchas innovaciones organizacionales $y$ ayuda a poner en marcha procesos participativos y colaborativos (semejantes a los de la economía colaborativa: Uber, Airbnb, Bla bla Car, Home Exchange, etc.) que no tienen precedentes en la historia reciente de nuestros países. Así se consolida una nueva forma, online, fácil y asequible de colaboración y participación política y ciudadana.

Este modelo posee ciertos elementos innovadores frente a otros ya existentes que describen a su vez formas de participación, de innovación y las consecuencias de todo ello en los procesos sociopolíticos (Ramírez-Alujas, 2010; García et alii, 2015).

El primer elemento del modelo es el empoderamiento individual y social para el cambio y la acción, tomando como base y estructura la denominada Teoría U principalmente desarrollada por Otto Scharmer y Katrin Kaufer (2013). Se puede decir que se trata de una teoría práctica que propone un método de presencia, es decir de atención plena que opera, cuando se practica, inevitablemente un cambio en individuos y grupos dado que comienzan a sentir y percibir que emerge el futuro desde ellos mismos; según los autores, el liderazgo en la actualidad debe partir de estas conceptualizaciones y además, su comprensión ayuda a desarrollar precisamente lo que denominamos autoliderazgo, cada individuo y grupo observan cómo llevan las riendas de sus vidas en todos los ámbitos. Los 
individuos en la socialización deben recibir herramientas que permitan desarrollar atributos de empoderamiento, como son, el desarrollo del autoliderazgo, la autoestima y el autocontrol emocional orientado todo al aumento de la resiliencia individual y social. Asimismo, se deben poseer conocimientos de finanzas responsables, derecho y pensamiento jurídico y finalmente ética social. Con lo cuál un número cada vez mayor de personas gozarán de la empatía, el equilibrio, solidaridad y capacidad de compasión y compromiso necesarios para alcanzar el objetivo participativo-colaborativo pretendido. Las instituciones educativas, desde el inicio, deben promover las herramientas básicas del auténtico trabajo en equipo, reparto de papeles y colaboración.

El segundo elemento del modelo es la propuesta del uso de redes sociales en Internet, pero yendo más allá tanto en el uso participativo y colaborativo que permiten las mismas como, en la utilización de las denominadas organizaciones incidentales; la idea es una apertura cuasi total de los temas a tratar y una búsqueda de soluciones que imite, en parte, a dichas organizaciones incidentales (Llano, 2017) que tienen lugar en las redes sociales, o bien en entornos físicos a través de convocatorias virtuales; se trata de encuentros casuales pero "relativamente organizados" en los cuáles grupos de gente muy numerosos y de forma espontánea participan a través de diferentes expresiones, bailes, paradas u otras para conseguir un determinado fin que puede ser tanto lúdico como social o incluso científico; es el caso de los Ice Bucket Challenge, Mannequin Challenge, The Harlem Shake, Flash Mobs, Ciencia ciudadana y otras experiencias similares que se plantean retos de todo tipo y que enganchan a todo el mundo, especialmente a la gente joven.

El tercer elemento son los procesos sociales indispensables para el correcto funcionamiento de las administraciones públicas, dispuestas al control de los problemas de agencia y orientadas al bien común; dichos procesos son: la participación ciudadana colaborativa y online (Gobierno abierto), la transparencia y la accountability. Se trata de aplicar las razones del humanismo empresarial (Llano Cifuentes, 1990) a las entidades públicas; además, de combinar diferentes perspectivas filosóficas que nos remiten al bien común y a la ética social. El modelo promueve procesos de innovación social que abren caminos para la creatividad social en todas sus dimensiones, con instituciones participativas y colaborativas y orientadas al buen gobierno. Un modelo basado en la confianza, aplicando el protocolo de la economía del bien común (Felber, 2012) y ofreciendo a asociaciones y organizaciones la posibilidad de trabajar desde y por la confianza.

Educación, empoderamiento, autoestima, libertad y pasión por lo que se hace son las bases del modelo que se propone y cuyas perspectivas, individuos/ grupos-objetivo y consecuencias son las que aparecen en la tabla 2. 
Tabla 2: Perspectivas, grupos-objetivo y consecuencias

\begin{tabular}{|c|c|c|}
\hline Perspectivas teóricas & Individuos/ Grupos-objetivo & $\begin{array}{l}\text { Consecuencias sociales, institu- } \\
\text { cionales e indicadores }\end{array}$ \\
\hline $\begin{array}{l}\text {-Perspectivas de orientación psi- } \\
\text { cosocial que desarrollan conceptos } \\
\text { como: autoliderazgo, motivación, } \\
\text { empoderamiento individual, gru- } \\
\text { pal y social. } \\
\text {-Teoría U } \\
\text {-Economía del Bien común }\end{array}$ & $\begin{array}{l}\text {-Escolares, } \\
\text {-Estudiantes de secundaria, } \\
\text {-Estudiantes universitarios } \\
\text {-Familias } \\
\text {-Asociaciones de barrios } \\
\text {-Otras asociaciones de la sociedad } \\
\text { civil } \\
\text {-Funcionarios } \\
\text {-Políticos y partidos políticos }\end{array}$ & $\begin{array}{l}\text {-Mayor compromiso de los funcio- } \\
\text { narios públicos. } \\
\text {-Mayor número de jóvenes ocupa- } \\
\text { dos en política } \\
\text {-Mayor número de información } \\
\text {-Mayores posibilidades de partici- } \\
\text { pación real y eficaz }\end{array}$ \\
\hline $\begin{array}{l}\text {-Aproximaciones filosóficas de } \\
\text { ética aplicada para la toma de de- } \\
\text { cisiones: Aristóteles, Kant, Rawls, } \\
\text { Cortina, Savater,... }\end{array}$ & $\begin{array}{l}\text {-Sociedad en su conjunto por que } \\
\text { la responsabilidad se distribuye de } \\
\text { forma equitativa. }\end{array}$ & $\begin{array}{l}\text {-Elevada motivación grupal, orga- } \\
\text { nizacional, sistémica y social } \\
\text {-Democratización real } \\
\text {-Juego limpio } \\
\text {-Aumento de la confianza social } \\
\text {-Relaciones y negociaciones de } \\
\text { suma no-cero. }\end{array}$ \\
\hline $\begin{array}{l}\text { Desarrollo de Big Data, Open Data } \\
\text { y TICs }\end{array}$ & $\begin{array}{l}\text { Participación ciudadana de manera } \\
\text { colectiva y colaborativa }\end{array}$ & $\begin{array}{l}\text { Mejora de las relaciones sociales, } \\
\text { generación de capital social e in- } \\
\text { telectual }\end{array}$ \\
\hline
\end{tabular}

Fuente: Elaboración propia

El modelo está basado en el uso de las tecnologías, se plasma en varias fases y posee los elementos indispensables para obtener una legitimación social máxima. La propuesta responde a la complejidad de la realidad sociopolítica actual pero aprovechando al máximo los logros alcanzados en relación a la cohesión social y al desarrollo socioeconómico consolidados en España. Las fases y elementos del modelo se resumen de la siguiente forma:

\section{Tabla 3. Fases y elementos de un ciclo del modelo de innovación sociopolítica}

Fase 1: Base común en la que se trabaja por grupos sociales heterogéneos la aplicación del cambio y empoderamiento individual y social en aplicación de la teoría $U$

Fase 2: Uso de redes sociales en Internet y gestión de Big Data y Open Data para ofrecer la información de los temas a debatir promoviendo la participación colaborativa y finalmente votar para la adopción de decisiones consensuadas socialmente

Fase 3: Promoción de grupos virtuales de análisis de las propuestas, con permiso de divulgación de sus datos, utilizando las mismas redes sociales. Generando debate y confrontación de ideas previas, siempre, a la toma de decisiones. Pueden ser los grupos establecidos trabajando con la teoría U

Fase 4: Propuesta de un calendario previo, conocido por toda la ciudadanía de cómo se realizará el planteamiento total de cualquiera de los temas sobre los que se tomarán decisiones, se señalarán las fases, se conocerán y divulgarán las resoluciones y cómo se seguirán finalmente las votaciones 
Fase 5: Creación de organizaciones incidentales, se organizan y plantean para la adopción de soluciones a problemas concretos y puntuales. Se pueden proponer a lo largo del tiempo de forma transversal

Fase 6: Para evitar los lobbies de intereses en determinados temas y que suelen acaparar la totalidad de votaciones, se fijará un mínimo de votos para poder sacar una propuesta adelante; si no, será necesario repetir la votación. Además, se realizarán preguntas en tiempo real que deberán ser respondidas por los ciudadanos con un lapso de tiempo determinado, no pudiendo repetir desde el mismo IP las respuestas

Fase 7: Voto, respuesta, propuestas electrónicas con un sistema de control que promueva la participación y el aseguramiento de la transparencia a lo largo de todo el proceso

Fase 8: Evaluación permanente, feedback e información ciudadana de los diferentes ciclos propuestos por temáticas, políticas, programas, etc. Cerrando un círculo informativo y de participacióncolaboración

Fuente: Elaboración propia

La consecuencia del modelo es tal que abre posibilidades de cambio social e institucional procurando un entorno en el que la participación activa, cualificada y productiva sea esencial. Ya no se trata de generar espacios asamblearios interminables, con egos incontrolables que desmontan cualquier posibilidad de eficacia en la toma de decisiones grupal. Las innovaciones tecnológicas abren la innovación institucional marcada por esa innovación social materializada en una participación virtual, colaborativa y altamente eficaz con redes sociales en Internet y un planteamiento maximizador y eficiente de los recursos. Todo ello debe venir acompañado de unos valores que son el fundamento sobre el que trabaja la teoría U y la teoría de la economía del bien común. Siendo los principales valores del modelo los siguientes: transparencia, confianza, colaboración, control de las acciones y propuestas por el propio sistema, solidaridad, mejora continua, democracia participativa y búsqueda del bien común. 


\section{Bibliografía}

Andreu Barberá, I., \& Brunet Icart, I. (2010). "La modernización administrativa en el Camp de Tarragona”. Revista de gestión pública y privada, (15), 7-30.

Bauman, Z. (2012). Daños colaterales: desigualdades sociales en la era global. Madrid:Fondo de Cultura Económica.

Brunet, I. y Rodríguez Soler, J. (2012) “Innovación y Formación profesional: Relaciones entre sistemas, políticas y actores". Barataria. Revista Castellano-Manchega de Ciencias Sociales, 14, 131-144.

Carbellido, V. M. N. (2005). ¿Qué es la calidad?: conceptos, gurús y modelos fundamentales. Buenos Aires:Limusa, Grupo Noriega Editores.

Castells, M. (2014). La crisis económica europea: una crisis política. Madrid:EuropeG. com.

Cervini, R. (2016). Corrupción política y sobrevivencia del estado democrático social de derecho. Madrid: Instituto de Derecho Penal.

Conteras, F. A. G.; Hidalgo, M. E. R.; Millán, A. G. L., \& Fernández, P. E. V. (2015)."Teoría de agencia (TA): supuestos teóricos aplicables a la gestión universitaria". Innovar: Revista de ciencias administrativas y sociales, 54,11-26.

Costa Cabanillas, E. y López Méndez, E. (2010). Los secretos de la dirección: Liderar y fortalecer personas y equipos. Madrid: Pirámide.

Cotino Hueso, L. (2015). "La nueva Ley de transparencia y acceso a la información". Anuario de la Facultad de Derecho, 7, 241-256.

Charron, Nicholas; Lewis Dijkstra and Victor Lapuente (2015).“Mapping the Regional Divide in Europe: A Measure for Assessing Quality of Government in 206 European Regions". Social Indicators Research. 122 (2), 315-346.

DiMaggio, P., y Powell, W. W. (1983). "The iron cage revisited: Collective rationality and institutional isomorphism in organizational fields". American Sociological Review, 48(2), 147-160.

Felber, C. (2012). La economía del bien común.Bilbao: Deusto SA Ediciones.

Fernández Dols, J. M. (1993). "Norma perversa: hipótesis teóricas”. Psicothema, 5, 91-101.

Fidone, G. (2011)."Eficacia y eficiencia en la reforma italiana de la administración pública (la llamada reforma Brunetta). De las medidas para la optimización de la productividad del empleo público a la «acción por la eficiencia»". Revista de Administración Pública, 186, 371-388.

García-Altés, A. y Argimon, J.M. (2016) "La transparencia en la toma de decisiones de salud pública”. Gaceta Sanitaria, 30, 9-13. 
García, C.; Carreón, J.; Mendoza, D;; Aguilar, J. A. y Hernández, J., J. (2015). "Contraste de un modelo de legitimidad sociopolítica". Tendencias y Retos, 20(2), 121-133.

Gobierno de España (2017) último acceso: julio 2017. <http:// transparencia.gob.es/transparencia/cavalencia/transparencia_Home/ index/GobiernoParticipacion/ParticipacionCiudadana/ParticipacionProye ctosNormativos/proyectoRDTransparencia.html>.

Gutiérrez David, E. (2014) «Derecho de acceso a la información pública». Eunomía, 6, 57-89.

Hölmsтrom, B. (1979). "Moral hazard and observability". The Bell journal of economics, 56, 74-91.

Husillos, J.; Larrinaga, C. y Álvarez, M.J. (2011). "La aparición y desarrollo de las memorias de sostenibilidad en España”. Revista Española de Financiación y Contabilidad, 124,195-219.

ITA (2017) Informe Transparencia Ayuntamientos. Transparencia Internacional. Última revisión: junio, 2017. Madrid.

Jensen, M. C., y Meckling, W. H. (1976). "Theory of the firm: Managerial behavior, agency costs and ownership structure". Journal of financial economics, 3(4), 305-360.

Jensen, M. C., \& Meckling, W. H.(1998). Divisional performance measurement. New York: Harvard University Press.

Keane, J. y Feenstra, R. A. (2014). Democracia monitorizada en España. Nuevas formas de participación politica en el marco de la era digital. Madrid: Alianza.

KPMG (2016). El camino hacia la transparencia y el buen gobierno en las Administraciones Públicas. Última revisión, julio 2017: kpmg.es.

Lizcaíno Álvarez, J. (2013)“Transparencia”. Eunomía, 3, 160-166.

López Laborda, J. y Rodrigo, F. (2014) "Los ciudadanos ante las haciendas regionales: quién es y quién debería ser responsable de servicios e impuestos". Revista de Economía Aplicada, 66, 5-33.

Llano-Cifuentes, A. (1999) "Actualidad del humanismo empresarial". Seminario permanente Empresa y Humanismo.Pamplona: Universidad de Navarra.

Llano, S. (2017) Organizaciones incidentales. Una revolución comunicativa. Madrid: AISOC.

Márquez, P. G. E. (2015)."Regeneración del Parlamento, transparencia y participación ciudadana". Teoría y realidad constitucional, (36), 171-194.

Mason, P. (2015) Postcapitalism. A guide to our future. London: Penguin Books. Moneva, J.M. (2007) “El marco de información sobre responsabilidad social de las organizaciones”. Ekonomiaz, 65, 285-317. 
Pérez Díaz, V. (1993). La primacía de la sociedad civil: el proceso de formación de la España democrática. Madrid:Alianza Editorial.

Pertuzé Fariña, C. (2015). "Nuevos tiempos para la gestión pública: potenciando la transparencia”. Revista Enfoques, 1(1), 19-27.

Ramírez-Alujas, A. V. (2010). "Innovación en la Gestión Pública y Open Government (Gobierno Abierto): Una Vieja Nueva Idea (Innovation in Public Management and Open Government: An Old New Idea)". Revista Buen Gobierno, 9, 214-231.

- (2011a). "Sobre la aplicación y desarrollo del concepto de innovación en el sector público: Estado del arte, alcances y perspectivas". Revista Circunstancia, $26,1-37$.

- (2011b). "Gobierno abierto y modernización de la gestión pública”. Revista Enfoques: Ciencia Política y Administración Pública, IX(15),99-125.

Requena, M. y Rodríguez, J. M. (2017). "Más allá de la democracia representativa: La democracia real y los movimientos sociales en el Estado español”. Revista Crítica de Ciências Sociais, (113), 3-28.

Ross, S. A. (1973). "The economic theory of agency: The principal's problem." The American Economic Review, 63(2), 134-139.

Sanagustín-Fons, M.V. (2011) Rural Tourism. A sustainable alternative. Applied Energy, Universidad de Zaragoza, vol. 80.

Sanagustín-Fons, M.V. (2011) Valores y ética empresarial. Un enfoque sociológico. Madrid: Trotta.

Schmitter, P. C., y Karl, T. L. (1991). "What democracy is... and is not". Journal of democracy, 2(3), 75-88.

Schmitter, P. C. (2004). "The ambiguous virtues of accountability". Journal of democracy, 15(4), 47-60.

Sсотт, W. R. (1995). Institutions and organizations. Foundations for organizational science. London: A Sage Publication Series.

Scharmer, O. y Kaufer, K. (2013). Leading from the Emerging Future. New York: Berrett-Koehler Publishers, Inc.

Schedler, A. (1999). Conceptualizing accountability. The self-restraining state: Power and accountability in new democracies. London, Boulder: Lynne Rienner Publishers.

Subirats, J. (Ed.). (1999)+ ¿Existe sociedad civil en España? responsabilidades colectivas y valores públicos. Madrid:Fundación Encuentro.

Subirats, J.; Alfama, E.; Obradors y Pineda, A. (2009) Ciudadania e inclusión social frente a las inseguridades contemporáneas. La significación del empleo. Documentos de Trabajo (Fundación Carolina), 32, 133-142. 
Swianiewicz, P. (2002) "Gobiernos locales en Europa Central y Oriental en una década de transformación". GAPP, Gestión y Análisis de Políticas Públicas, 25, 67-95.

Wheeler, D., y Sillanpä̈̈, M. (1997). The stakeholder corporation: A blueprint for maximizing stakeholder value. London:Pitman.

Williamson, O. E. (2005). "The economics of governance". The American Economic Review, 95(2), 1-18. 